Journal for ImmunoTherapy of Cancer

\title{
Humoral and cellular correlates of a novel immune-related adverse event and its treatment
}

\author{
Amrit S Gonugunta (D) , ${ }^{1}$ Mitchell S von Itzstein, ${ }^{2,3}$ Hong Mu-Mosley, ${ }^{3}$ \\ Farjana Fattah, ${ }^{3} \mathrm{~J}$ David Farrar, ${ }^{3,4}$ Angela Mobely, ${ }^{4}$ Sawsan Rashdan, ${ }^{2,3}$ Sunny Lai, ${ }^{2}$ \\ Salman F Bhai, ${ }^{5}$ Bonnie L Bermas, ${ }^{3,6}$ David Karp, ${ }^{3,6}$ Quan-Zhen Li, ${ }^{4,6}$ \\ Edward K Wakeland, ${ }^{4}$ David E Gerber ${ }^{2,3,7}$
}

To cite: Gonugunta AS, von Itzstein MS, Mu-Mosley H, et al. Humoral and cellular correlates of a novel immune-related adverse event and its treatment. Journal for ImmunoTherapy of Cancer 2021;9:e003585. doi:10.1136/jitc-2021-003585

- Additional supplemental material is published online only. To view, please visit the journal online (http://dx.doi.org/10. 1136/jitc-2021-003585).

ASG and MSvl contributed equally.

Accepted 04 November 2021

Check for updates

(C) Author(s) (or their employer(s)) 2021. Re-use permitted under CC BY-NC. No commercial re-use. See rights and permissions. Published by BMJ.

For numbered affiliations see end of article.

\section{Correspondence to} Dr David E Gerber; David.Gerber@utsouthwestern. edu

\section{ABSTRACT}

Immune-related adverse events (irAE) may affect almost any organ system and occur at any point during treatment with immune checkpoint inhibitors (ICI). We present a patient with advanced lung cancer receiving antiprogrammed death 1 checkpoint inhibitor who developed a delayed-onset visual irAE treated with corticosteroids. Through assessment of longitudinal biospecimens, we analyzed serial autoantibodies, cytokines, and cellular populations. Months after ICI initiation and preceding clinical toxicity, the patient developed broad increases in cytokines (most notably interleukin-6 (IL-6), interferon- $\gamma$ (IFN $\gamma)$, C-X-C motif chemokine ligand 2 (CXCL2), and C-C motif chemokine ligand 17 (CCL17)), autoantibodies (including antiangiotensin receptor, $\alpha$-actin, and amyloid), CD8 T cells, and plasmablasts. Such changes were not observed in healthy controls and ICl-treated patients without irAE. Administration of corticosteroids resulted in immediate and profound decreases in cytokines, autoantibodies, and inflammatory cells. This case highlights the potential for late-onset changes in humoral and cellular immunity in patients receiving $\mathrm{ICl}$. It also demonstrates the biologic effects of corticosteroids on these parameters. Application of humoral and cellular immune biomarkers across ICI populations may inform toxicity monitoring and management.

While immune checkpoint inhibitors (ICI) have revolutionized the treatment of cancer, associated toxicities termed immune-related adverse events (irAE) occur in a substantial proportion of patients. These autoimmune events may affect almost any organ system. Although irAE tend to occur within the first 3 months of ICI therapy, they may occur at almost any point during treatment. ${ }^{12}$ When irAE occur, the dose and duration of corticosteroids and other immunosuppressants remain somewhat arbitrary.

We present a case of late-onset visual irAE. Serial assessment of humoral and cellular immune parameters identifies potential explanations for irAE timing as well as biologic effects of steroids.

\section{PATIENT, MATERIALS, AND METHODS} Clinical data and sample collection

The patient was enrolled in a prospective biospecimen collection protocol approved by the UT Southwestern IRB (\#STU 082 015-053). The patient provided written, informed consent for additional data collection, presentation, and publication. Clinical, radiographic, and laboratory data were collected from the Epic electronic health record (Epic, Verona, Wisconsin).

Peripheral blood samples were examined from the patient and two cases without irAE (no toxicity (NT)) at pre-ICI baseline, 2 weeks, 6 weeks, 3 months, every 3 months thereafter, and after initiation of steroids for irAE treatment. We also examined serial samples (baseline, 2 weeks) from two healthy controls. Samples were centrifuged at $3000 \mathrm{rpm}$ at $4^{\circ} \mathrm{C}$ for $15 \mathrm{~min}$ to obtain plasma. Peripheral blood mononuclear cells (PBMCs) were isolated from samples using density gradient centrifugation in Ficoll-Paque Plus Media (Fisher Scientific, Waltham, Massachusetts).

\section{Cytokine/chemokine analysis}

As previously described, monitoring of cytokine and chemokine levels was performed using Bio-Plex Pro Human Chemokine 40-plex Panel (Bio-Rad Laboratories, Hercules, California) using a Luminex 200 System. Bio-Plex Manager 6.1 software was used for data analysis. Concentrations of cytokines and chemokines $(\mathrm{pg} / \mathrm{mL})$ were determined on the basis of the fit of a standard curve for mean fluorescence intensity versus $\mathrm{pg} / \mathrm{mL}$. These cytokines are stable over time in healthy controls not receiving 
ICI. ${ }^{3}$ Heatmaps were generated using Genesis cluster analysis of microarray data. ${ }^{4}$

\section{Autoantigen array analysis}

As we have described previously, serum autoantibody profiling was performed using a custom protein array panel of 124 autoantigens, including nuclear antigens, cytosolic/matrix antigens, and tissue/organ-specific antigens. ${ }^{56}$ Data analysis included the following preprocessing steps: (1) background subtraction and averaging of duplicated spots; (2) normalization of the signal intensity of each Ag using internal controls across all samples; and (3) normalized signal intensity (NSI) for each Ag (Ab) completed per sample. NSI files were processed for downstream analysis using the Cluster and Treeview algorithm. Heatmaps were generated using Genesis cluster analysis of microarray data. ${ }^{4}$

\section{Cytometry by time of flight (CyTOF)}

Cryopreserved PBMCs were thawed and stained with a panel of 36 antibodies (metal isotope-labeled conjugates, Maxpar Direct Immune Profiling Assay Panel by Fluidigm). Cells were analyzed on a Helios mass cytometer (Fluidigm). Data were normalized and analyzed with gating on CD45+ cells using a cloud-based computational platform OMIQ.ai (Omiq, Santa Clara, California). Equal sampling of 7669 events per sample (lowest common denominator across samples) and a total of 176387 events across all 23 samples were analyzed using uniform manifold approximation and projection (UMAP) clustering. We analyzed distribution and expression characteristics of phenotypic markers using EdgR at OMIQ on the abundance of the 41 clusters between the patient and the control samples. We identified cluster immune phenotypes following standard immunophenotyping for the Human Immunology Project. ${ }^{7}$

\section{RESULTS}

\section{Clinical case}

A 56-year-old woman with a 30 pack-year smoking history presented with hemoptysis. She had a history of chronic obstructive pulmonary disease, hypertension, and dyslipidemia, but no autoimmune, vascular, or neurologic disease. Imaging demonstrated a $1.7 \mathrm{~cm}$ right middle lobe mass, a subcentimeter left parietal brain metastasis, and multiple pleural deposits. Biopsy demonstrated poorly differentiated squamous cell carcinoma with programmed death-ligand 1 staining greater than $50 \%$.

The patient received single-agent pembrolizumab, which was tolerated well and resulted in prolonged disease control. Almost 1 year after treatment initiation, she developed blurry vision in the left eye without headache or temporal artery tenderness. On examination, she had 20/200 vision in the left eye and 20/20 vision in the right, an afferent pupillary defect, and severe left optic disc edema. Laboratory analysis was remarkable for $\mathrm{C}$ reactive protein of $88 \mathrm{mg} / \mathrm{L}$ (reference range $\leq 5 \mathrm{mg} / \mathrm{L}$ ).
MRI of the orbits showed no enhancement of orbits or optic nerves. Temporal artery biopsy did not demonstrate active or healed arteritis.

The patient received methylprednisolone $1 \mathrm{~g}$ intravenous daily for presumed arteritic ischemic optic neuropathy, with mild subjective improvement in vision, then discharged with a slow prednisone taper starting at $60 \mathrm{mg}$ orally daily. More than 1 year after the event, the patient remained off any systemic cancer therapy with ongoing disease control but only modest improvement was observed in visual acuity (online supplemental figure 1).

\section{Immune parameters}

Figure 1A,B displays serial cytokine and autoantibody determinations. In general, levels of selected cytokines demonstrated modest increases at the 3-month and 6-month timepoints, but substantial changes did not occur until 9 months after ICI initiation. In particular, proinflammatory cytokines and chemokines such as IL-6, IL-1 $\beta$, TNF $\alpha$, IFN $\gamma$, CXCL2, and CCL17 exhibited clear increases at that timepoint in the patient but not in the NT patients treated with ICI. We previously demonstrated longitudinal stability of these parameters in the healthy control patients. ${ }^{3}$ Similarly, autoantibody levels broadly increased after initiation of ICI. Antibodies against angiotensin receptor (AGTR), $\alpha$-actin, U1-snRNP A, amyloid, and collagen II exhibited the greatest increases in the patient in comparison to the NT cases. Following initiation of high-dose corticosteroids (12-month timepoint), levels of cytokines and autoantibodies broadly and promptly decreased.

We compared longitudinal immune cell profiling in peripheral blood samples from the patient (seven timepoints), two NT cases (seven timepoints), and two healthy controls (two timepoints) using high-dimensional mass cytometry (CyTOF). We identified 41 clusters representing B cells, CD4/CD8 T cells, monocytes, and NK cells (figure 2A,B). We identified 13 distinctive clusters with statistical significance $(q<0.01)$ between patient and control samples (figure 2C and D). Compared with healthy controls, patients with cancer had significantly lower naive and memory B cells (figure 2D and E). Compared with other cases, the patient had significantly lower naive CD4, CD8, and gamma-delta $\mathrm{T}$ cells (figure 2D and F, online supplemental figure 2). At later timepoints (3, 6 , and 9 months; figure 2D), we noted increased subsets of effector memory and terminal effector CD8 T cells, plasmablasts, and CD14+CD27-B cells in the patient compared with the NT cases and healthy controls.

\section{DISCUSSION}

Despite years of research and ICI clinical trials, the optimal approach to monitoring and treating irAE remains unknown. Ocular toxicity, which developed in the presented case almost 1 year after ICI initiation, is an established but rare $(<1 \%)$ event associated with ICI. The blood-retinal barrier, lack of efferent lymphatics, and 

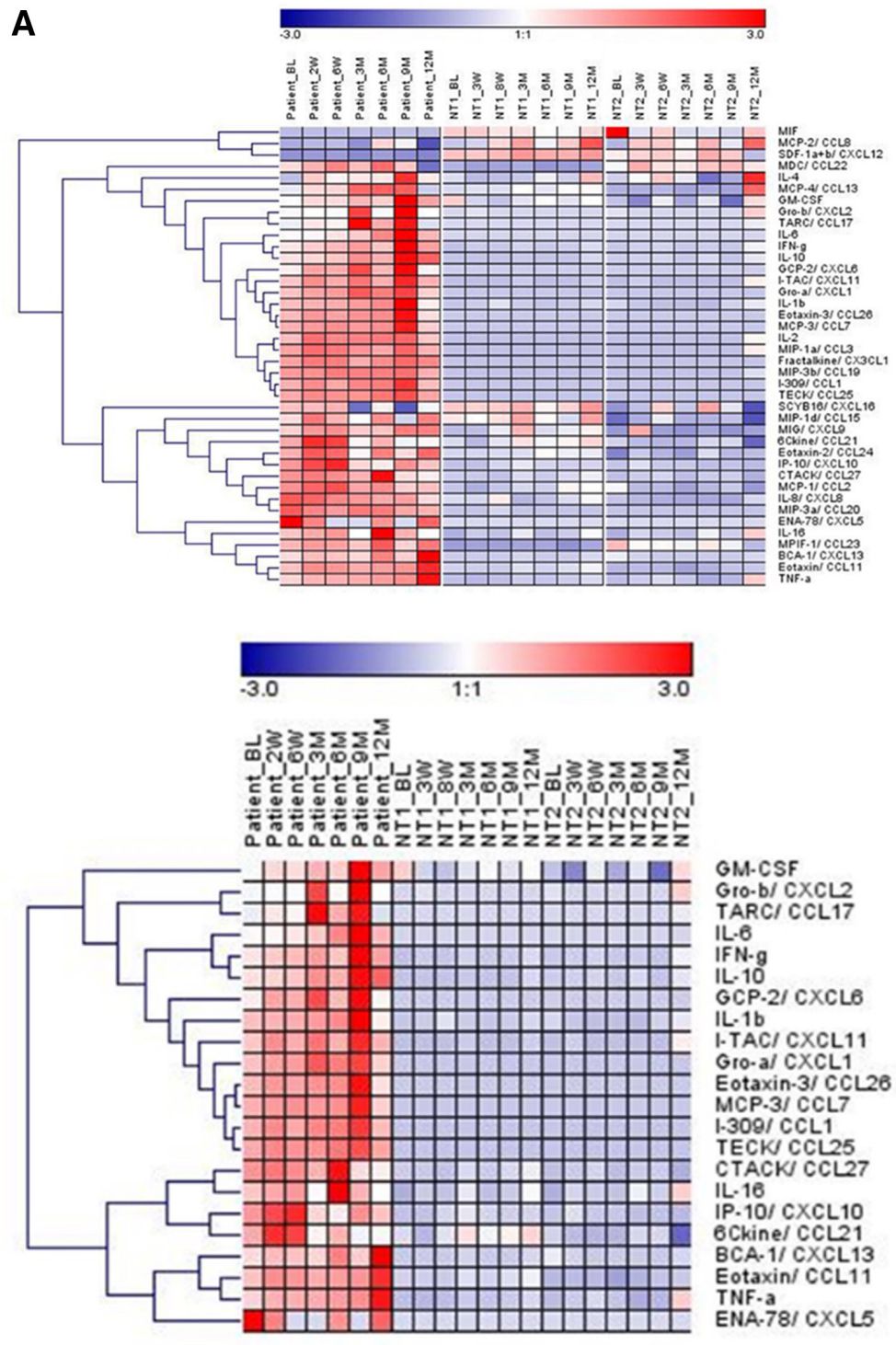
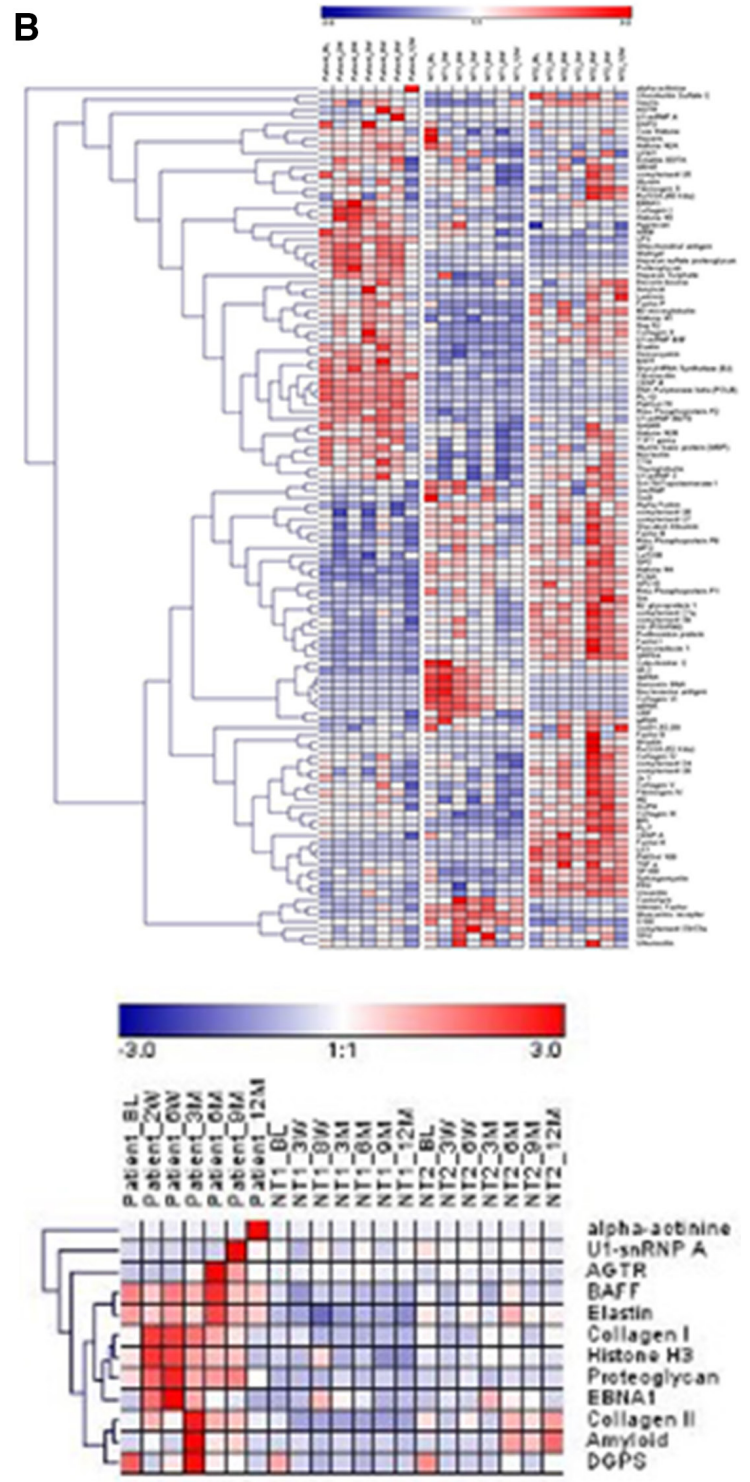

Figure 1 Heatmaps of cytokines (A) and antibodies (B) across timepoints with comparison to the no toxicity (NT) cases. Both full panels (upper) and elevated panels specific to the patient (bottom) are shown. Heatmaps from healthy controls have been previously published. ${ }^{3} \mathrm{BL}$, baseline.

upregulation of cytokines such as Fas-L and TNF $\beta$ render the orbit an immune privileged structure. ${ }^{8}$ Among these cases, uveitis, episcleritis, and blepharitis are the most common manifestations. ${ }^{9} 10$ To our knowledge, the present case represents the first description of apparent ICI-associated arteritic ischemic optic neuropathy. While late irAE occur less frequently than early toxicities, they are relatively common in long-term responders such as the patient described here. ${ }^{2}$

Our analysis of systemic immune parameters revealed a broad but modest increase shortly after ICI initiation. A more profound change occurred 9 months after treatment initiation, an event that may explain the delayedonset irAE. Among cytokines, IL6, IL1 $\beta$, TNF $\alpha$, CXCL2, IFN $\gamma$, and CCL17, which are involved in the proinflammatory cascade, demonstrated particular increases. Prior to onset of patient's visual symptoms, numerous antibodies increased substantially, including AGTR, which is expressed on endothelial cells and pericytes, is associated with vasoconstriction, and may be implicated in neurovascular ophthalmic disorders. ${ }^{11}$

Accompanying these cytokine and autoantibody derangements, we observed significant increases in terminal effector CD8 T cells, plasmablasts, and CD14+ $\mathrm{B}$ cells in the patient compared with the NT and healthy controls, particularly after 3 months of ICI therapy. Immune cell populations were relatively stable over time in the healthy and NT controls, although we recognize the limitations of examining only a 2-week interval in the healthy controls. Additionally, we noted that-compared with healthy controls-the patients with cancer in this analysis were deficient in both CD8+ T cells and B cells. Whether this reflects effects of malignancy, prior cancer treatment, or a chance finding requires further study. 
A

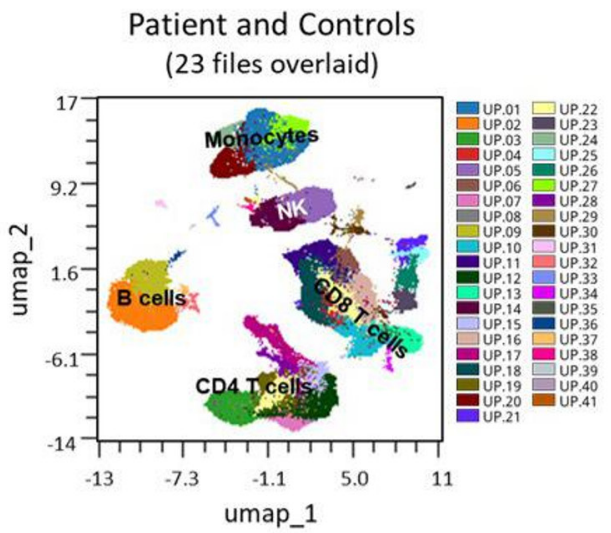

C

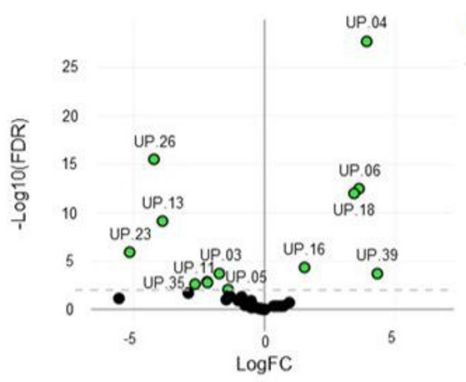

B

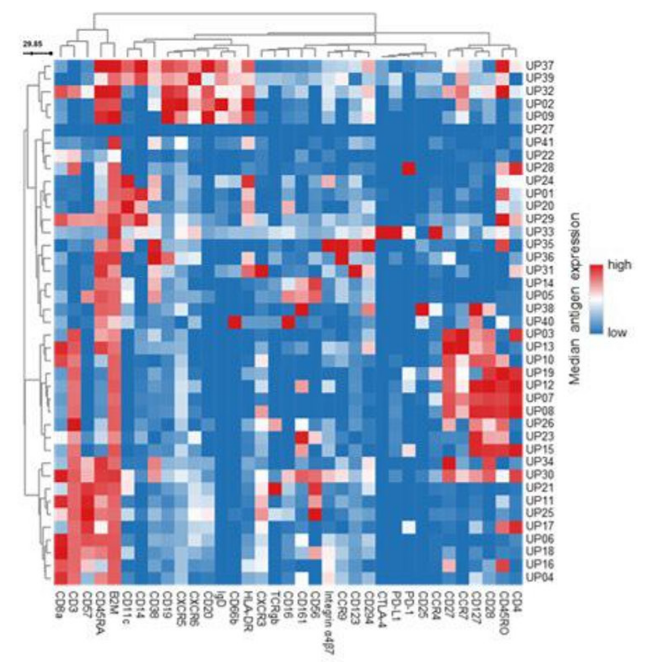

D

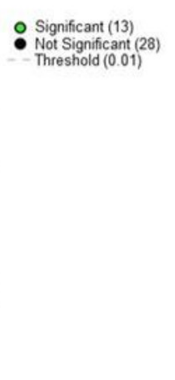

$\mathbf{E}$

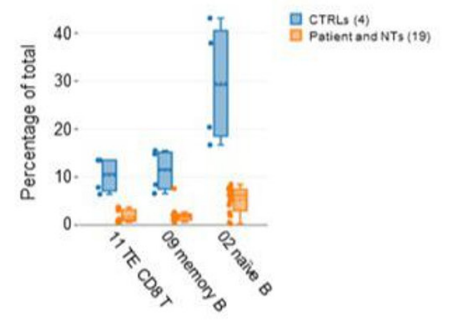

$\mathbf{F}$

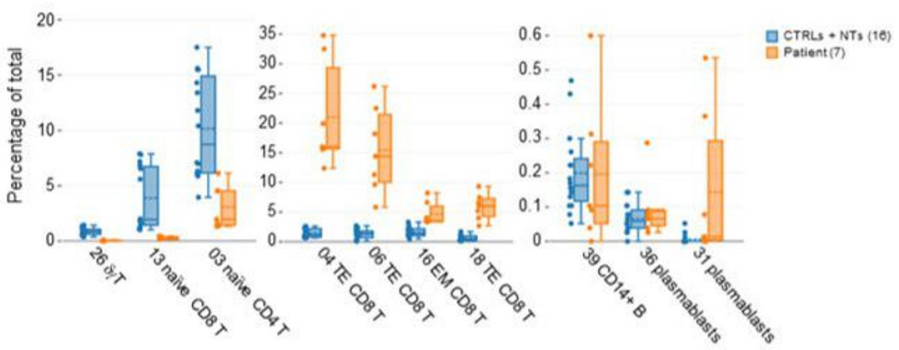

Figure 2 Immune cell profiles of the patient, no toxicity (NT) cases, and healthy controls in peripheral blood by cytometry by time of flight. (A) A total 41 clusters were identified in all 23 files corresponding to B cells, CD4 T cells, CD8 T cells, monocytes, and NK cells. Colored dots in UMAP plot represent cell events. (B) Heatmap of surface marker expression in each cluster on patient baseline sample. The median antigen expression is shown in the heatmap. (C) Volcano plot shows clusters with significant differences between the patient, NT cases, and healthy controls (CTRL) by EdgR statistical analysis. Significant clusters are shown in green, $q<0.01$. (D) Heatmap of clusters with changes among all timepoints from patient, NT cases, and healthy controls. The abundance of cell events per cluster is shown. Box charts show differences in clusters between patients with cancer (with and without irAE) and healthy controls $(\mathrm{E})$ and differences between the patient and NT cases+healthy controls (F). The percentage of the total event count per cluster is shown. Solid lines designate median; dotted lines designate mean. Bars represent maximum and minimum values. Dots adjacent to boxes represent individual samples. irAE, immune-related adverse events; NK, natural killer; UMAP, uniform manifold approximation and projection.

The treatment of irAE is largely empirical. For clinically significant events, expert guidelines often recommend initiating relatively high-dose corticosteroids with slow tapers. ${ }^{12}$ Despite this aggressive (and potentially toxic) approach, some patients have refractory or recurrent irAE, eventually requiring alternative immunosuppressive agents. Conversely, other patients might benefit from far lower doses and shorter tapers. Laboratory parameters indicating near-term physiologic effects in patients receiving steroids for irAE treatment might allow a more personalized approach to toxicity management. With serial biospecimens from before and after steroid initiation in the present case, we were able to observe immediate and profound suppression across all immune parameters. Determining whether such changes correlate with the degree and duration of clinical benefit will require further study. 
Author affiliations

${ }^{1}$ Medical School, University of Texas Southwestern Medical Center, Dallas, Texas, USA

${ }^{2}$ Department of Internal Medicine (Division of Hematology and Oncology), University of Texas Southwestern Medical Center, Dallas, Texas, USA

${ }^{3}$ Harold C. Simmons Comprehensive Cancer Center, University of Texas

Southwestern Medical Center, Dallas, Texas, USA

${ }^{4}$ Department of Immunology (Division of Microarray and Immune Phenotyping Core Facility), University of Texas Southwestern Medical Center, Dallas, Texas, USA

${ }^{5}$ Department of Neurology, University of Texas Southwestern Medical Center, Dallas, Texas, USA

${ }^{6}$ Department of Internal Medicine (Division of Rheumatology), University of Texas Southwestern Medical Center, Dallas, Texas, USA

${ }^{7}$ Department of Population and Data Sciences, University of Texas Southwestern Medical Center, Dallas, Texas, USA

Contributors ASG and MSvl contributed equally for the production of this manuscript.

Funding This work was supported in part by a National Cancer Institute Midcareer Investigator Award in Patient-Oriented Research (K24 CA201543-01), the National Institute of Allergy and Infectious Disease (1U01Al156189-01), an American Cancer Society-Melanoma Research Alliance Team Award (MRAT-18-114-01-LIB), a V Foundation Robin Roberts Cancer Survivorship Award (DT2019-007), the University of Texas Lung Cancer Specialized Program of Research Excellence (P50CA070907-21), and a UT Southwestern Burroughs Wellcome Fund PhysicianScientist Institutional Award. This work was also supported by the Human Genomics/Microarray Core and Biomarker Research Core at the Harold C. Simmons Comprehensive Cancer Center (2P30 CA142543-01).

Competing interests None declared.

Patient consent for publication Consent obtained directly from patient(s).

Provenance and peer review Not commissioned; externally peer reviewed.

Supplemental material This content has been supplied by the author(s). It has not been vetted by BMJ Publishing Group Limited (BMJ) and may not have been peer-reviewed. Any opinions or recommendations discussed are solely those of the author(s) and are not endorsed by BMJ. BMJ disclaims all liability and responsibility arising from any reliance placed on the content. Where the content includes any translated material, BMJ does not warrant the accuracy and reliability of the translations (including but not limited to local regulations, clinical guidelines, terminology, drug names and drug dosages), and is not responsible for any error and/or omissions arising from translation and adaptation or otherwise.
Open access This is an open access article distributed in accordance with the Creative Commons Attribution Non Commercial (CC BY-NC 4.0) license, which permits others to distribute, remix, adapt, build upon this work non-commercially, and license their derivative works on different terms, provided the original work is properly cited, appropriate credit is given, any changes made indicated, and the use is non-commercial. See http://creativecommons.org/licenses/by-nc/4.0/.

ORCID iD

Amrit S Gonugunta http://orcid.org/0000-0002-1666-9871

\section{REFERENCES}

1 Sosa A, Lopez Cadena E, Simon Olive C, et al. Clinical assessment of immune-related adverse events. Ther Adv Med Oncol 2018;10:175883591876462

2 Nigro O, Pinotti G, De Galitiis F, et al. Late immune-related adverse events in long-term responders to PD-1/PD-L1 checkpoint inhibitors: a multicentre study. Eur J Cancer 2020;134:19-28.

3 Khan S, Khan SA, Luo X, et al. Immune dysregulation in cancer patients developing immune-related adverse events. $\mathrm{Br} J$ Cancer 2019;120:63-8.

4 Sturn A, Quackenbush J, Trajanoski Z. Genesis: cluster analysis of microarray data. Bioinformatics 2002;18:207-8.

$5 \mathrm{Li} \mathrm{Q}-Z$, Zhou J, Wandstrat AE, et al. Protein array autoantibody profiles for insights into systemic lupus erythematosus and incomplete lupus syndromes. Clin Exp Immunol 2007;147:60-70.

6 Khan S, von Itzstein MS, Lu R, et al. Late-Onset immunotherapy toxicity and delayed autoantibody changes: checkpoint inhibitorinduced Raynaud's-Like phenomenon. Oncologist 2020;25:e753-7.

7 Maecker HT, McCoy JP, Nussenblatt R. Standardizing immunophenotyping for the human immunology project. Nat Rev Immunol 2012;12:191-200.

8 Della Vittoria Scarpati G, Fusciello C, Perri F, et al. Ipilimumab in the treatment of metastatic melanoma: management of adverse events. Onco Targets Ther 2014;7:203-9.

9 Brahmer JR, Lacchetti C, Schneider BJ, et al. Management of immune-related adverse events in patients treated with immune checkpoint inhibitor therapy: American Society of clinical oncology clinical practice guideline. J Clin Oncol 2018;36:1714-68.

10 Fang T, Maberley DA, Etminan M. Ocular adverse events with immune checkpoint inhibitors. J Curr Ophthalmol 2019;31:319-22.

11 Clermont A, Bursell S-E, Feener EP. Role of the angiotensin II type 1 receptor in the pathogenesis of diabetic retinopathy: effects of blood pressure control and beyond. J Hypertens Supp/ 2006;24:S73-80.

12 Brahmer JR, Abu-Sbeih H, Ascierto PA, et al. Society for immunotherapy of cancer (SITC) clinical practice guideline on immune checkpoint inhibitor-related adverse events. J Immunother Cancer 2021;9:e002435. 